\title{
Regulation of brain-derived neurotrophic factor (BDNF) in the chronic unpredictable stress rat model and the effects of chronic antidepressant treatment
}

\author{
Marianne H. Larsen ${ }^{\text {a,b,* }}$, Jens D. Mikkelsen ${ }^{\text {a }}$, Anders Hay-Schmidt ${ }^{\text {, }}$, Carmen Sandi ${ }^{\text {b }}$ \\ ${ }^{a}$ Department of Neuroscience and Pharmacology, University of Copenhagen, Denmark \\ ${ }^{\mathrm{b}}$ Brain Mind Institute, École Polytechnique Fédérale de Lausanne, Switzerland
}

\section{A R T I C L E I N F O}

\section{Article history:}

Received 16 October 2009

Received in revised form 8 January 2010

Accepted 12 January 2010

\section{Keywords:}

Chronic unpredictable stress

Depression

Antidepressants

Brain-derived neurotrophic factor

Hippocampus

Anhedonia

\begin{abstract}
A B S T R A C T
Chronic unpredictable stress (CUS) is a widely used animal model of depression. The present study was undertaken to investigate behavioral, physiological and molecular effects of CUS and/or chronic antidepressant treatment (venlafaxine or imipramine) in the same set of animals. Anhedonia, a core symptom of depression, was assessed by measuring consumption of a palatable solution. Exposure to CUS reduced intake of a palatable solution and this effect was prevented by chronic antidepressant treatment. Moreover, chronic antidepressant treatment decreased depressive-like behavior in a modified forced swim test in stressed rats. Present evidence suggests a role for brain-derived neurotrophic factor (BDNF) in depression. BDNF mRNA levels in the ventral and dorsal hippocampus were assessed by in situ hybridization. Exposure to CUS was not correlated with a decrease but rather with an increase in BDNF mRNA expression in both the dentate gyrus of the dorsal hippocampus and the CA3 region of the ventral hippocampus indicating that there is no simple link between depression-like behaviors per se and brain BDNF levels in rats. However, a significant increase in BDNF mRNA levels in the dentate gyrus of the dorsal hippocampus correlated with chronic antidepressant treatment emphasizing a role for BDNF in the mechanisms underlying antidepressant activity.
\end{abstract}

(c) 2010 Elsevier Ltd. All rights reserved.

\section{Introduction}

Depression is a severe disorder that has enormous consequences for the individual's quality of life, and it is among the most prevalent forms of mental illness. Clinical symptoms like depressed mood, anhedonia, fatigue or loss of energy, feelings of worthlessness or guilt, and diminished ability to concentrate or think are characteristic for depression. Despite the devastating impact of depression, relatively little is known about the etiology and pathogenesis of depression.

Today, most effective antidepressants work through modulation of serotonergic and noradrenergic neurotransmission. However, increasing evidence indicates that neurotrophins such as brain-derived neurotrophic factor (BDNF) may play a role in the pathophysiology of depression and that antidepressants may in part exert their effects through regulation of BDNF. Several clinical studies have reported that serum BDNF levels are decreased in depressed patients, and that they can be normalized by antidepressant treatment (Aydemir et al., 2005, 2006; Brunoni et al., 2008; Gervasoni et al., 2005; Gonul et al., 2005). Moreover, genetic stud-

\footnotetext{
* Corresponding author. Address: Department of Neuroscience and Pharmacology, University of Copenhagen, Blegdamsvej 3, 2200 Copenhagen N, Denmark. Tel.: +45 3532 7264; fax: +453532 7610 .

E-mail address: haldlarsen@hotmail.com (M.H. Larsen).
}

ies indicate that polymorphisms in the BDNF gene (Val66Met) may be associated with increased susceptibility to develop major depression (Aguilera et al., 2009; Schumacher et al., 2005; Wichers et al., 2008).

Stress is known to be an important factor in the etiology of depression and several animal studies have reported that exposure of rats to stress can result in decreased hippocampal BDNF levels (Nibuya et al., 1999; Rasmusson et al., 2002; Smith et al., 1995). Conversely, chronic antidepressant treatment, as well as repeated electroconvulsive seizures, was reported to enhance BDNF expression in the rat hippocampus (Larsen et al., 2005, 2008; Nibuya et al., 1995; Rogoz et al., 2005; Sillaber et al., 2008). In fact, antidepressant activity seems to be dependent on normal BDNF function (Monteggia et al., 2004, 2007; Saarelainen et al., 2003), and infusion of BDNF into either the midbrain or the hippocampus was shown to induce antidepressant-like effects in behavioral models of depression (Shirayama et al., 2002; Siuciak et al., 1997), suggesting that BDNF exhibits antidepressant activity in its own.

The precise neuroanatomical basis of depression is still poorly understood, but strong evidence indicates that the hippocampus is one of the brain regions that play a central role in many aspects of depression (Drevets et al., 2008; Duman and Monteggia, 2006). BDNF is highly expressed in the hippocampus and most studies investigating the role of BDNF in depression have focused on the expression of BDNF in the dorsal hippocampus. It is important to 
note that whereas the dorsal part of the hippocampus has been strongly implicated in memory function (Bannerman et al., 2004; Moser et al., 1993), the ventral part was shown to be involved in regulation of the stress response and defensive and fear-related behaviors (Kjelstrup et al., 2002; Trivedi and Coover, 2004). Anxiety and fear are symptoms that are often present in depressed individuals and the ventral hippocampus may thus play an equally important role in depression.

The chronic unpredictable stress (CUS) model is an animal model of depression which is considered to have a greater face validity to model this disorder than other animal models (Willner, 1997). In the CUS paradigm, rats are exposed to a variety of different unpredictable stressors, which consequently lead to development of depressive-like behaviors such as increased anhedonic behavior (a core symptom of depression) (Willner, 1997, 2005).

To advance our understanding of the role of BDNF in the pathophysiology and treatment of depression, we here study both behavioral and molecular alterations induced by CUS in concert with the effects of chronic antidepressant treatment. We have previously shown that chronic treatment with the antidepressants, imipramine and venlafaxine, induced a robust increase in BDNF mRNA expression in the dorsal hippocampus whereas no effect was observed after chronic treatment with the selective serotonin reuptake inhibitor (SSRI), fluoxetine (Larsen et al., 2008). To the best of our knowledge, this is the first study to report the effects of CUS and chronic treatment with two different antidepressants, imipramine and venlafaxine, on depressive-like behavior as well as on the expression of BDNF mRNA in both the dorsal and ventral rat hippocampus in the same set of animals.

\section{Materials and methods}

\subsection{Animals}

Adult male Sprague-Dawley rats (Charles River Laboratories) (200-250 g) were housed 2 per cage separated by a transparent plastic separator in standard plastic cages $\left(1177 \mathrm{~cm}^{2}\right.$ surface area). The rats were housed under light- and temperature-controlled conditions with free access to food and water. The rats were allowed to acclimatize to these conditions for 7 days before any experimental procedure was initiated. A total number of 56 rats were used in the present study. All experimental procedures were approved by the Swiss Cantonal Veterinary Office Committee for Animal Experimentation and were carried out in accordance with the Declaration of Helsinki, the European Communities Council Directive of 24 November 1986 (86/609/EEC) and with the Swiss National Institutional Guidelines on Animal experimentation.

\subsection{General experimental procedure}

Before start of the CUS protocol the animals were tested in the elevated plus maze (EPM) and in the open field and novel object $(\mathrm{OF} / \mathrm{NO})$ test to determine the individual anxiety level. It is known that the individual response to stress exposure is variable and development of depression may indeed be associated with the individual anxiety trait (Sandi and Richter-Levin, 2009). To account for any anxiety trait factor, the animals were matched according to their anxiety level and their body weight to ensure that the same anxiety level and body weigh distribution was obtained in each group. Subsequently the groups were randomly assigned to one of the following experimental groups ( $n=8$ per group):

(1) Daily treatment with saline ( $5 \mathrm{ml} / \mathrm{kg}$, i.p.).

(2) Daily treatment with venlafaxine $(10 \mathrm{mg} / \mathrm{kg}$, i.p.).

(3) Daily treatment with imipramine (10 $\mathrm{mg} / \mathrm{kg}$, i.p.).
(4) Chronic unpredictable stress and daily treatment with saline ( $5 \mathrm{ml} / \mathrm{kg}$, i.p.).

(5) Chronic unpredictable stress and daily treatment with venlafaxine ( $10 \mathrm{mg} / \mathrm{kg}$, i.p.).

(6) Chronic unpredictable stress and daily treatment with imipramine (10 $\mathrm{mg} / \mathrm{kg}$, i.p.).

Behavioral indexes of the impact of CUS and antidepressant treatment were evaluated through a saccharin consumption test performed on day 11,18 and 25 of the CUS protocol and a forced swimming test performed towards the end of the CUS procedure (on day 27 of the CUS protocol). The body weight was measured the day before starting the stress-induction protocol and then every 3rd day throughout the stress period.

\subsection{Elevated plus maze}

Anxiety-related behaviors were evaluated using the elevated plus maze (EPM) test (Herrero et al., 2006). Briefly, the plus maze consists of two opposing open arms $(45 \times 10 \mathrm{~cm})$ and two enclosed arms $(45 \times 10 \times 38 \mathrm{~cm})$ that extend from a central platform $(10 \times 10 \mathrm{~cm})$, elevated $50 \mathrm{~cm}$ above the ground. The light was adjusted to levels of 10-12 lx in the center of the maze. The rats were placed individually on the central platform and allowed to explore the maze for $5 \mathrm{~min}$. Behavior was monitored using a video camera and analyzed with a computerized tracking system (Ethovision 3.1.16, Noldus IT, The Netherlands). Time spent in the open and closed arms (and their edges) was recorded. The anxiety level was assessed by the time spent in the open arm. Rats were assigned to the different experimental groups according to the time they spent in the open arm and the groups were balanced with regard to the same parameter.

\subsection{Open field and novel object}

Anxiety-related behavior was tested in the open field (OF) and novel object (NO) test. The open field consisted of a black pool with a diameter of $1 \mathrm{~m}$ and a depth of $40 \mathrm{~cm}$. The floor of the pool was divided into three zones: outer zone with a diameter of $1 \mathrm{~m}$, inner zone with a diameter of $75 \mathrm{~cm}$, and the center zone with a diameter of $25 \mathrm{~cm}$. The light was adjusted to a level of $8-10 \mathrm{~lx}$ in the center of the pool. Animals were placed in the center of the pool and the open field activity was tested for a $10 \mathrm{~min}$ period. Subsequently a novel object was introduced into the center of the pool, and the behavior was observed during the following $5 \mathrm{~min}$. The activity and behavior during the whole session was recorded with a video camera, and the time spent in each of the zones was automatically registered and analyzed with the computerized tracking system EthoVision (Color-Pro 3.0.15, Nodulus Information Technology, The Netherlands). Behavioral analysis was done manually and included: time freezing and the time touching the novel object. The anxiety level was assessed by the time spent in the center zone and the time touching the novel object. The rats were assigned to the different experimental groups according to the time they spent in the center zone and the time touching the novel object, and the groups were balanced with regard to the same parameters.

\subsection{Chronic unpredictable stress}

In conformity with previously published CUS protocol (Sandi et al., 2008), rats were each day subjected to one of nine stressors in an unpredictable order and at an unpredictable time of the day for a period of 28 days (Table 1 ). The stressors included acoustic stimulation (78-115 dB noise bursts), inverse light and dark cycle (over a $48 \mathrm{~h}$ period), exposure to overcrowding under a bright light 
Table 1

Schematic presentation of CUS schedule.

\begin{tabular}{|c|c|}
\hline Day of treatment & Stressor used \\
\hline Day 1 & Acoustic stimulation \\
\hline Day 2 & Inverse light dark cycle \\
\hline Day 3 & Inverse light dark cycle \\
\hline Day 4 & Bright light \& overcrowding \\
\hline Day 5 & Foot shock \\
\hline Day 6 & Elevated platform \\
\hline Day 7 & Predator odor \\
\hline Day 8 & Bright light \& overcrowding \\
\hline Day 9 & Elevated platform \\
\hline Day 10 & Food deprivation \\
\hline Day 11 & Foot shock ${ }^{\mathrm{a}}$ \\
\hline Day 12 & Predator odor \\
\hline Day 13 & Inverse light dark cycle \\
\hline Day 14 & Inverse light dark cycle \\
\hline Day 15 & Bright light \& overcrowding \\
\hline Day 16 & Bright light \& water deprivation \\
\hline Day 17 & Elevated platform \\
\hline Day 18 & Foot shock ${ }^{\mathrm{a}}$ \\
\hline Day 19 & Predator odor \\
\hline Day 20 & Bright light \& overcrowding \\
\hline Day 21 & Food deprivation \\
\hline Day 22 & Elevated platform \\
\hline Day 23 & Bright light \& water deprivation \\
\hline Day 24 & Acoustic stimulation \\
\hline Day 25 & Foot shock ${ }^{\mathrm{a}}$ \\
\hline Day 26 & Predator odor \\
\hline Day 27 & Forced swim \\
\hline Day 28 & Forced swim \\
\hline Day 29 & Sacrifice \\
\hline
\end{tabular}

a Days where saccharin test was performed.

(six rats in a standard home cage, $1000 \mathrm{~lx}, 2 \mathrm{~h}$ ), inescapable foot shock (three foot shocks of $1 \mathrm{~mA}, 1 \mathrm{~s}$ ), elevated platform (rats were placed on a platform $(20 \times 20 \mathrm{~cm})$ elevated $1 \mathrm{~m}$ above the ground for $2 \mathrm{~h}$ ), predator odor ( $1 \mathrm{~h}$ exposure to 2,5-dihydro-2,4,5-trimethylthiazoline, a synthetic compound originally isolated from fox feces (Fendt and Endres, 2008)), bright light and water deprivation (1000 lx, $30 \mathrm{~min}$ ), food deprivation (24 h), and forced swim stress (15 min). Non-stressed control rats were housed under similar conditions but left undisturbed during the stress period.

\subsection{Modified forced swim test}

A modified (as it was used as one of the stressors in the CUS protocol) forced swim test was included as a stressor on day 27 in the CUS protocol. The animal's behavior was monitored to evaluate potential differences between the stressed animals submitted to different drug treatments. In brief, rats were individually forced to swim for $15 \mathrm{~min}$ in a plastic tube (25 $\mathrm{cm}$ in diameter, $46 \mathrm{~cm}$ deep) containing $30 \mathrm{~cm}$ of temperate water $\left(25^{\circ} \mathrm{C} \pm 1\right)$. Behavior was recorded with a video camera during the 15 min session, and the time spent floating, swimming, climbing and diving was recorded manually.

\subsection{Saccharin consumption and preference test}

A saccharin consumption test was performed on day 11,18 and 25 of the stress regime. A naïve unchallenged group (no stress and no treatment) $(n=8)$ was also tested in order to obtain a baseline measurement. The rats were given a free choice between two bottles, one containing normal drinking water and the other containing $0.02 \%$ saccharin solution. The bottles were left for $12 \mathrm{~h}$. The saccharin intake was calculated as the amount consumed in gram per $100 \mathrm{~g}$ body weight. The saccharin preference was calculated as saccharin intake/total fluid intake (water + saccharin).

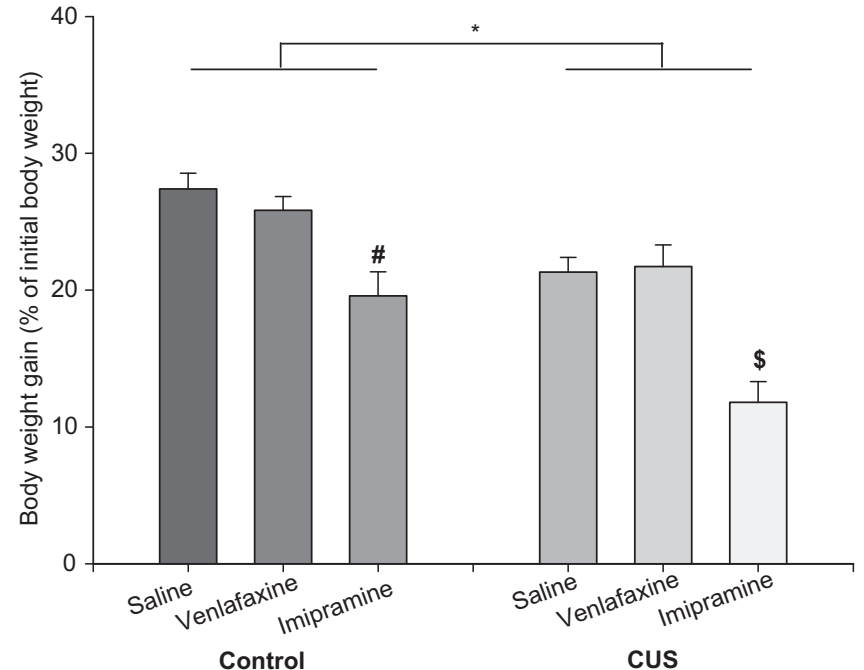

Fig. 1. Exposure to CUS for 28 days attenuated body weight gain. The increase in body weight is calculated as the \% increase of initial body weight. Results are presented as mean \pm SEM. ${ }^{*} P<0.05 ;{ }^{\#} P<0.05$ compared to control-saline; ${ }^{\$} P<0.05$ compared to CUS-saline (two-way ANOVA followed by Fishers LSD post hoc test).

\subsection{Drug treatments}

The experimental groups were randomly assigned to treatment with saline $(5 \mathrm{ml} / \mathrm{kg})$, venlafaxine $(10 \mathrm{mg} / \mathrm{kg})$ or imipramine $(10 \mathrm{mg} / \mathrm{kg}$, Sigma). Drugs were dissolved in saline and were injected intraperitoneally (i.p.) in a volume of $5 \mathrm{ml} / \mathrm{kg}$. Animals were injected daily in the morning (7:30-9 a.m.) for 28 consecutive days starting on the same day as the CUS procedure.

\subsection{Tissue processing and in situ hybridization}

Rats were decapitated $24 \mathrm{~h}$ after the last drug treatment. Brains were quickly removed, frozen on dry ice and stored at $-80^{\circ} \mathrm{C}$ until further processed for in situ hybridization. Consecutive coronal sections were collected from the dorsal hippocampus ( -3.60 until -4.16 relative to bregma) and from the ventral hippocampus ( -4.8 until -5.20 relative to bregma) where the ventral CA3 region is clearly identified but before appearance of the ventral dentate gyrus (Paxinos and Watson, 1998). Briefly, sections were acetylated (10 min in $0.25 \%$ acetic anhydride (Merck KgaA, Darmstadt, Germany), 0.1 M triethanolamine (Sigma-Aldrich), $0.9 \% \mathrm{NaCl}, \mathrm{pH}$ 8.0) followed by delipidation and dehydration in a series of ethanol solutions (70\%/5 min, 80\%/1 min, 95\%/2 min and 99\%/1 min). Finally, the sections were incubated for $5 \mathrm{~min}$ in chloroform (Merck, Germany). Excessive chloroform was washed off the slides in $99 \%$ and $96 \%$ ethanol, and the slides were allowed to air-dry. Sections were hybridized with synthetic oligonucleotides (DNA Technology A/S, Denmark) specific for rat mRNA encoding BDNF (bases 586630; accession: NM_012513). The probe was 3'-tail labeled with $\alpha-\left[{ }^{35} \mathrm{~S}\right] \mathrm{dATP}(>3000 \mathrm{Ci} / \mathrm{mmol}$, GE Healthcare, UK). The labeled probe was added at a specific activity of $1 \times 10^{6} \mathrm{cpm} / 100 \mu \mathrm{l}$ to the hybridization buffer containing $45 \%$ formamide $(\mathrm{v} / \mathrm{v}), 4 \times$ saline sodium citrate (SSC) $(1 \times$ SSC is $0.15 \mathrm{M} \mathrm{NaCl}, 0.015 \mathrm{M} \mathrm{NaCi}$ trate $\left.2 \mathrm{H}_{2} \mathrm{O}, \mathrm{pH} 7.2\right), 1 \times$ Denhardts solution (0.02\% ficoll, polyvinylpyrrolidone and bovine serum albumin) (Sigma), fish sperm DNA $(0.5 \mathrm{mg} / \mathrm{ml})$ (Roche Molecular Biochemicals A/S, Denmark), $0.25 \mathrm{mg} / \mathrm{ml}$ yeast t-RNA (Sigma-Aldrich), 10\% (w/v) dextran sulphate (Sigma-Aldrich) and $10 \mathrm{mM}$ dithiothreitol (Sigma-Aldrich). After overnight incubation with $100 \mu$ of the hybridization mixture/slide $\left(37^{\circ} \mathrm{C}\right)$ the slides were transferred to four rapid consecutive washes in $1 \times$ SSC (room temperature) and then washed four 
(A)

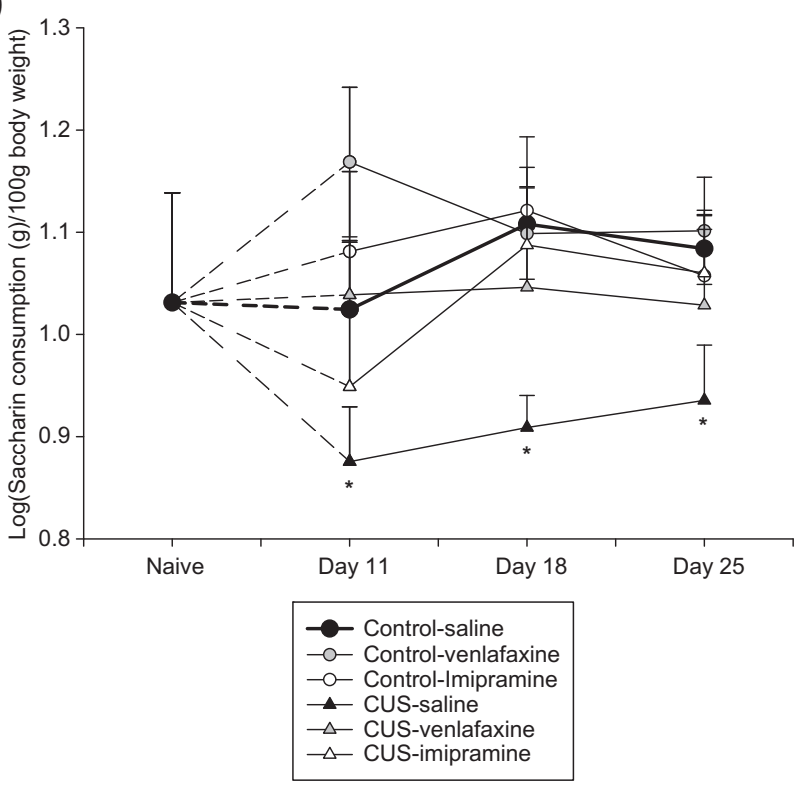

(B)

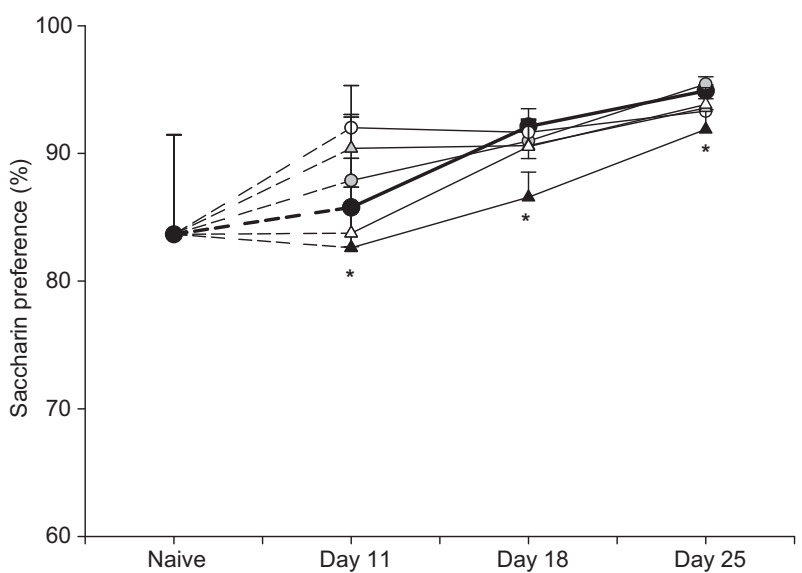

Fig. 2. Saccharin consumption (A) and saccharin preference (B) was measured after 11,18 and 25 days of CUS. Baseline for both parameters was assessed in a group of naïve unchallenged rats. Saccharin consumption per $100 \mathrm{~g}$ body weight (A) and saccharin preference (B) was significantly decreased in rats exposed to CUS-saline as compared to the control-saline group. Imipramine and venlafaxine treatment prevented CUS induced reduction in saccharin consumption and saccharin preference as compared to control-saline treated rats. Results for saccharin consumption have been log-transformed in order to obtain equal variance. Results are presented as mean \pm SEM. ${ }^{*} P<0.05$ compared to control-saline (one-way repeated ANOVA).

times for $15 \mathrm{~min}$ in $1 \times \operatorname{SSC}\left(55^{\circ} \mathrm{C}\right)$ and two times for $30 \mathrm{~min}$ in $1 \times$ SSC at room temperature. Excessive salt was washed of the slides in DEPC-treated water. Finally, the sections were dried and exposed together with ${ }^{14} \mathrm{C}$ standards (Amersham Bioscience, England) to a Kodak BiomaxMR film (GE Healthcare) for 2-18 days depending on the expression level of the gene. Films were developed using an AGFA Curix 60 developer (AGFA, Germany). High resolution scans were obtained using a Molecular Imager GS-800 scanner (Bio-Rad Laboratories Inc., Herlev, Denmark). Optical densities were quantified using the image analysis system, Quantity One 1-D Analysis Software version 4.5.2 (Bio-Rad Laboratories Inc., Hercules, CA). Gray density values were measured in the area of interest (Fig. 4) on three to four sections from each animal and the values were calibrated to the ${ }^{14} \mathrm{C}$-tissue standards. Gray density values were measured in a representative area on each slide and the value was subtracted from each of the measurements. No signal was detected by BDNF oligonucleotide sense probe.

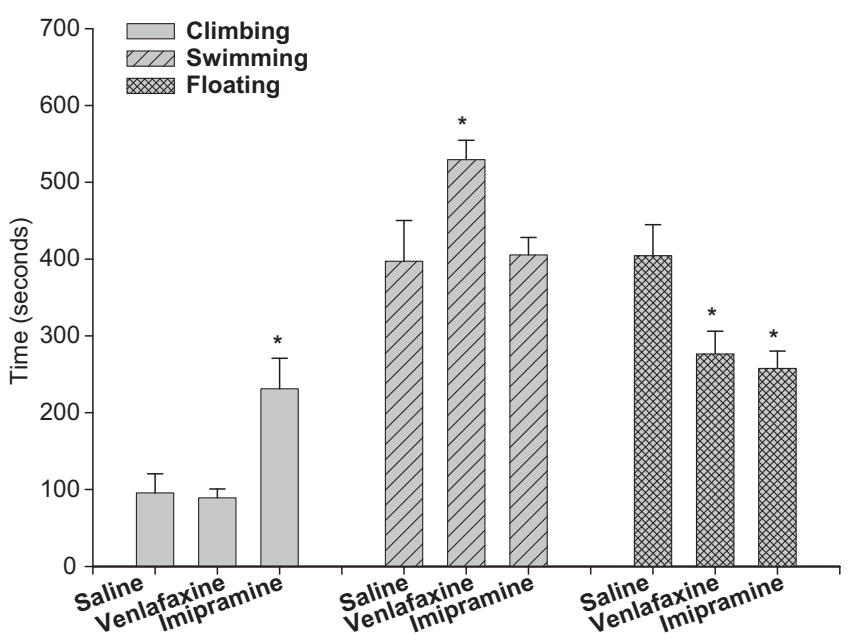

Fig. 3. Depressive-like behavior after 27 days of CUS was evaluated in a $15 \mathrm{~min}$ forced swim test. Imipramine and venlafaxine treatment significantly decreased the time spent floating as compared to saline treated rats. An increase in climbing behavior was observed in rats treated with imipramine and an increase in swimming behavior was observed in rats treated with venlafaxine as compared to saline treated rats. Results are presented as mean \pm SEM. $\stackrel{*}{P}<0.05$ compared to saline treatment (one-way ANOVA followed by Fishers LSD post hoc test).

\subsection{Statistics}

Statistical analyses were performed with SPSS 13.0 (LEAD Technologies Inc.). Data were analyzed using, as appropriate, two-way ANOVA (treatment $\times$ stress as factors) followed by Fishers LSD post hoc test or a one-way ANOVA followed by Fishers LSD post hoc test. Saccharin data were analyzed using a two-way repeated ANOVA analysis with treatment and stress as between subject factors and time as within subject factor. In addition to the general comparisons, we were interested in analyzing the respective effects of stress and antidepressant treatment by direct comparison of the results induced by each of these treatments to the control-saline group. In these cases, each repeated ANOVA analysis was followed by Fishers LSD post hoc test. Correlation analyses were performed using the Pearson's correlation test. Statistical significance was accepted at $P<0.05$.

\section{Results}

Before exposure to CUS, all rats were tested in the EPM and OF/ NO test and the anxiety level for each rat was determined. The experimental groups were balanced with regard to anxiety level and body weight (Table 2 ).

\subsection{Effects of CUS on body weight and saccharin intake}

The impact of CUS was first evaluated by examining body weight gain during the 28 days of CUS and the effect on preference for a sweet solution after 11,18 and 25 days of exposure to the stress protocol. All experimental groups were equivalent in their body weight prior to the onset of CUS. Rats exposed to 28 days of CUS showed significantly lower body weight gain than control animals (\% gain (mean $\pm \mathrm{SEM}$ ): control-saline, $27.4 \pm 1.2$; control-venlafaxine, $25.8 \pm 1.0$; control-imipramine, $19.6 \pm 1.8$; CUS-saline, $21.3 \pm$ 1.1; CUS-venlafaxine, $21.8 \pm 1.6$; CUS-imipramine, $11.8 \pm 1.5$ ). A two-way ANOVA analysis revealed a significant main effect of CUS $(F(1,46)=29.38 ; P<0.001)$ as well as a significant main effect of antidepressant treatment $(F(2,46)=25.04 ; P<0.001)$ on body weight gain. No interaction between CUS and antidepressant treatment was found $(F(2,46)=0.86$, n.s.). Subsequent post hoc analysis 
Table 2

Anxiety levels and body weight for each experiment group before exposure to CUS.

\begin{tabular}{|c|c|c|c|c|c|c|}
\hline & Control-saline & CUS-saline & Control-venlafaxine & CUS-venlafaxine & Control-imipramine & CUS-imipramine \\
\hline EPM (\% time in open arm) & $37.5 \pm 9.3$ & $30.5 \pm 6.9$ & $22.0 \pm 4.1$ & $20.7 \pm 4.3$ & $29.2 \pm 2.9$ & $25.3 \pm 3.5$ \\
\hline OF (\% time in center) & $4.8 \pm 0.8$ & $4.3 \pm 0.6$ & $3.9 \pm 0.5$ & $4.7 \pm 0.8$ & $4.2 \pm 0.4$ & $4.2 \pm 0.5$ \\
\hline NO (\% time touching novel object) & $22.9 \pm 2.8$ & $22.7 \pm 3.3$ & $20.9 \pm 3.6$ & $22.5 \pm 4.7$ & $24.4 \pm 2.4$ & $21.0 \pm 3.4$ \\
\hline Body weight $(\mathrm{g})$ & $364.0 \pm 4.8$ & $368.5 \pm 5.2$ & $363.0 \pm 5.9$ & $372.4 \pm 7.1$ & $365.8 \pm 6.9$ & $372.5 \pm 6.7$ \\
\hline
\end{tabular}

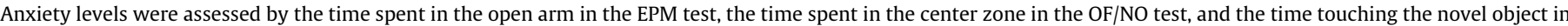
the OF/NO test. No differences between any of the groups in any of the parameters were observed. Data are expressed as mean \pm SEM.

showed that the effect of treatment was due to a significant reduction in body weight gain after chronic treatment with imipramine whereas venlafaxine had no effect on the body weight gain as compared to the respective saline treated groups (Fig. 1).

Saccharin consumption was measured after 11, 18 and 25 days of exposure to CUS (Table 1). An overall comparison revealed that rats exposed to CUS drank significantly less of the saccharin solution per $100 \mathrm{~g}$ body weight as compared to control rats (two-way repeated ANOVA), $F(1,43)=5.34 ; P<0.05$ ) (Fig. 2 A). There was no significant main effect of antidepressant treatment $(F(2,43)=1.72$; n.s. $)$ or time $(F(2,86)=2.10$; n.s. $)$ and no significant interaction between CUS and antidepressant treatment $(F(2,43)=0.52$; n.s. $)$, between CUS and time $(F(2,86)=1.37$; n.s. $)$, or between treatment and time $(F(4,86)=1.50$; n.s.). Further repeated ANOVA analyses between the control-saline group and the other groups (with time as repeated factor), revealed that only CUS-saline treated rats drank significantly less of the saccharin solution as compared to the control-saline group $(F(1,16)=8.61$; $P<0.05)$. There was no effect of time $(F(2,32)=1.49$; n.s. $)$ and no interaction between time and $\operatorname{CUS}(F(2,32)=0.32$; n.s.). No differences between CUS-imipramine and control-saline $(F(1,15)=0.19$; n.s.) or between CUS-venlafaxine and control-saline $(F(1,15)=$ 0.15 ; n.s.) were observed. In line with this, saccharin consumption was unaffected by drug treatment in the non-stressed control groups (Fig. 2A).

When the saccharin preference index was evaluated, a trend towards a significant main effect of stress on saccharin preference was observed (two-way repeated ANOVA, $F(1,44)=2.82 ; P=0.1$ ). No effect of antidepressant treatment was found $(F(2,44)=0.95$; n.s.), but a significant effect of time was observed $(F(2,88)=13.80 ; P<0.05)$. There was no significant interaction between CUS and antidepressant treatment $(F(2,44)=1.05$; n.s.), between CUS and time $(F(2,88)=0.20)$, or between antidepressant treatment and time $(F(4,88)=0.33$; n.s.). Further repeated ANOVA analyses between the control-saline group and each of the other groups (with time as repeated factor) only revealed a significant difference in saccharin preference between CUS-saline and control-saline $(F(1,17)=5.24 ; P<0.05)$ (Fig. 2B).

\subsection{Chronic antidepressant treatment decreases depressive-like behavior in the forced swim test}

Only animals exposed to the CUS regime were exposed to the forced swim test as this test was employed as a stressor on day 27 in the CUS protocol. In the forced swim test, floating behavior is interpreted as a measure of depressive-like behavior which can be reduced by antidepressant treatment (Porsolt et al., 1978). As expected, a significant effect of antidepressant treatment on floating behavior was found (one-way ANOVA, $F(2,21)=6.38$; $P=0.007)$. Post hoc analysis revealed that animals treated with imipramine or venlafaxine spent significantly less time floating as compared to saline treated animals during the $15 \mathrm{~min}$ swim test (Fig. 3). In addition, antidepressant treatment had significant effects on climbing behavior (one-way ANOVA, $F(2,21)=8.24$; $P=0.002$ ) as well as on swimming behavior (one-way ANOVA, $F(2,21)=4,15 ; P=0.03)$. Subsequent post hoc analysis revealed that rats treated with imipramine spent significantly more time climbing compared to saline treated rats, whereas venlafaxine had no effect on the climbing behavior. In contrast, rats treated with venlafaxine spent significantly more time swimming compared to saline treated rats, whereas no effect was observed in rats treated with imipramine (Fig. 3).

\subsection{Effects of antidepressant treatment and CUS on BDNF mRNA expression in the hippocampus}

The effects of antidepressant treatment on BDNF mRNA levels were determined by densitometric analysis of BDNF mRNA expression in the granular cell layer (GCL) of the dentate gyrus in the dorsal hippocampus and in the CA3 region of the dorsal and ventral hippocampus (Fig. 4). As reported previously, the levels of BDNF are higher in the GCL and in the CA3 region as compared to the CA1 region, and the expression levels were only quantified in regions where the expression levels were clearly distinguishable from background levels (Larsen et al., 2008).

In the dorsal hippocampus, overall ANOVA revealed that BDNF mRNA levels were significantly increased in the GCL in CUS-exposed animals as compared to controls (two-way ANOVA, $F(1,45)=11.95$; $P<0.001$ ) (Fig. 5A). An overall effect of antidepressant treatment was found (two-way ANOVA, $F(2,45)=4.01 ; P=0.025$ ) and post hoc analysis confirmed an effect of venlafaxine and imipramine on BDNF mRNA expression in the GCL as compared to saline treatment. Venlafaxine induced BDNF mRNA expression with $14 \%$ in the CUS group and with $14 \%$ in the control group as compared to their respective saline treated groups. Imipramine treatment resulted in a 15\% increase in the CUS group and 16\% increase in the control group as compared to the respective saline treated groups (Fig. 5A). No interaction between CUS and antidepressant treatment was found $(F(2,45)=0.02$; n.s.). Conversely, neither CUS nor chronic antidepressant treatment had effects on the BDNF expression in the CA3 region of the dorsal hippocampus and no interaction between CUS and chronic antidepressant treatment was found (CUS: $F(1,45)=0.68$, n.s.; antidepressant: $F(2,45)=3.07$, n.s.; CUS $\times$ antidepressant: $F(2,45)=0.39$, n.s.) (Fig. 5B).

In the ventral hippocampus, BDNF mRNA expression levels were measured in the CA3 region. An overall significant increase in BDNF mRNA expression in the CA3 region of the ventral hippocampus was observed in animals exposed to CUS as compared to non-stressed animals (two-way ANOVA, $F(1,45)=6.52$; $P=0.014)$. Chronic treatment with venlafaxine or imipramine had no effect on BDNF mRNA expression in the CA3 region of the ventral hippocampus as compared to saline treatment (two-way ANOVA, $F(2,45)=0.96$; n.s.) and no significant interaction between CUS and antidepressant treatment was found (two-way ANOVA, $F(2,45)=0.592$; n.s.) (Fig. 6) .

\subsection{Correlations between basal anxiety levels and post-treatment BDNF MRNA levels}

No correlation was found between basal anxiety levels (as evaluated before treatments started and indexed by parameters such as \% time in EPM open arm, \% time in OF center, \% time touching 


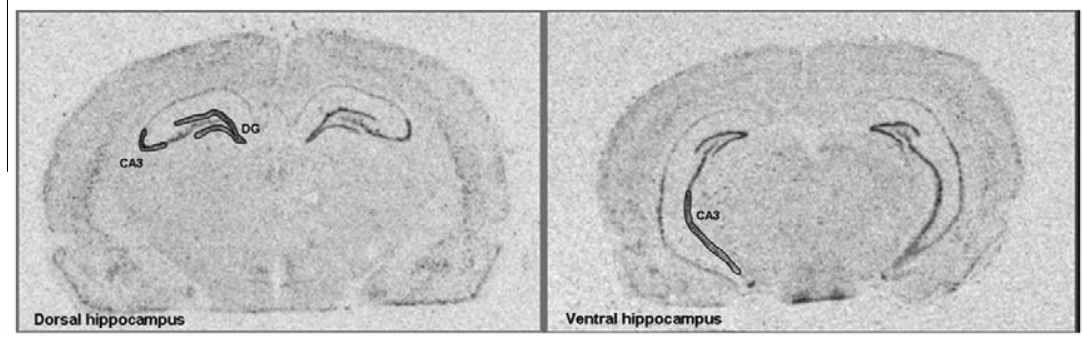

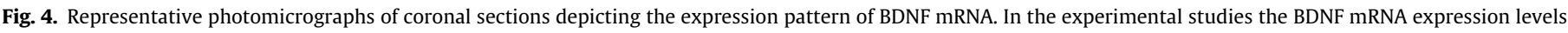

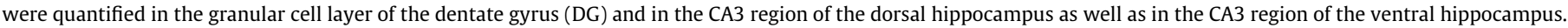
The areas of interest are outlined on the photomicrographs.

(A)
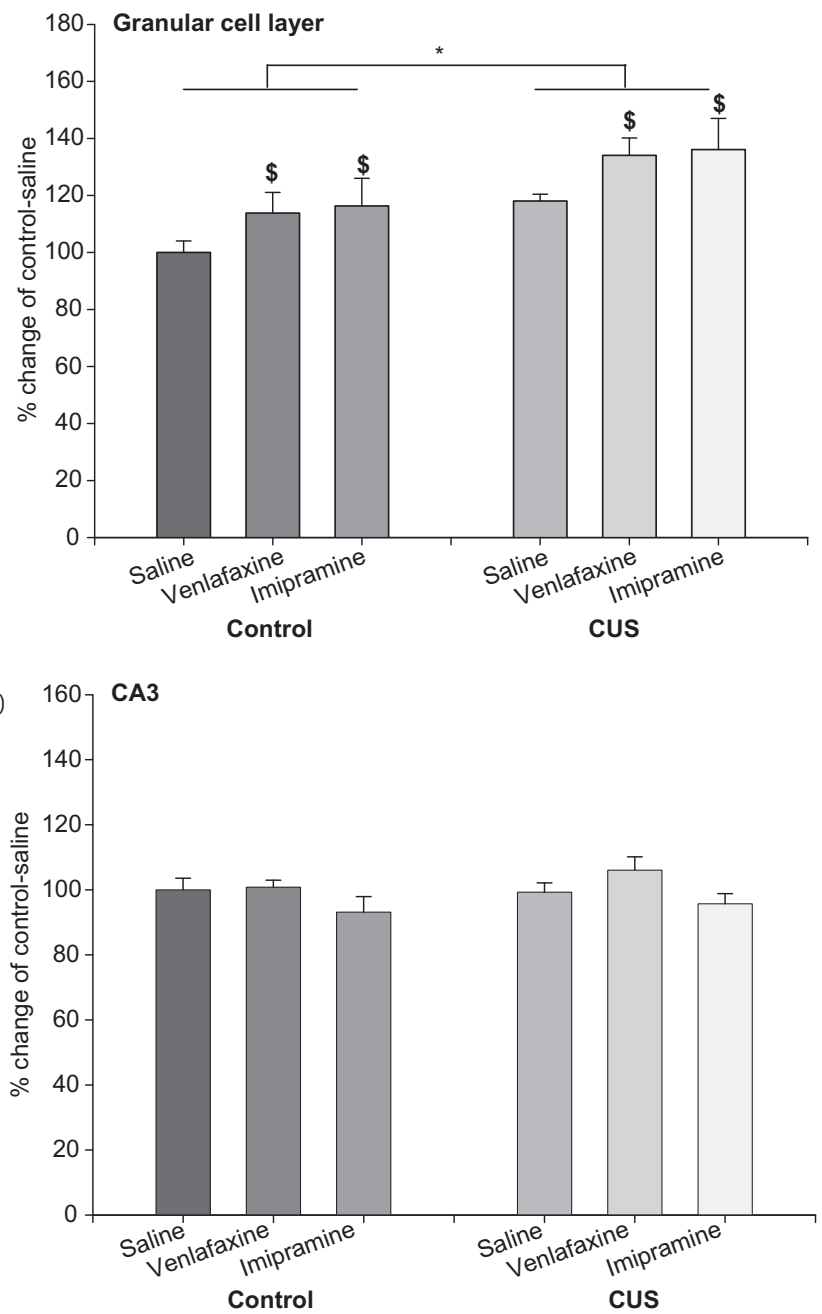

Fig. 5. Effects of CUS and chronic treatment with venlafaxine or imipramine on BDNF mRNA levels in the GCL (A) and the CA3 (B) region of the dorsal hippocampus. An overall significant increase in BDNF mRNA levels in the GCL was observed in animals exposed to CUS as compared to control animals (A). An overall effect of venlafaxine and imipramine on BDNF mRNA expression in the GCL was found as compared to saline treatment (A). BDNF mRNA levels, in the CA3 region, were not affected by CUS or by chronic antidepressant treatment (B). Results are presented as mean \pm SEM. ${ }^{*} P<0.05 ;{ }^{\$} P<0.05$ compared to saline within the group (two-way ANOVA followed by Fishers LSD post hoc test).

NO) and BDNF mRNA expression in any of the hippocampal subregions analyzed (i.e., the dorsal dentate gyrus, dorsal CA3 or ventral CA3 regions) (data not shown).

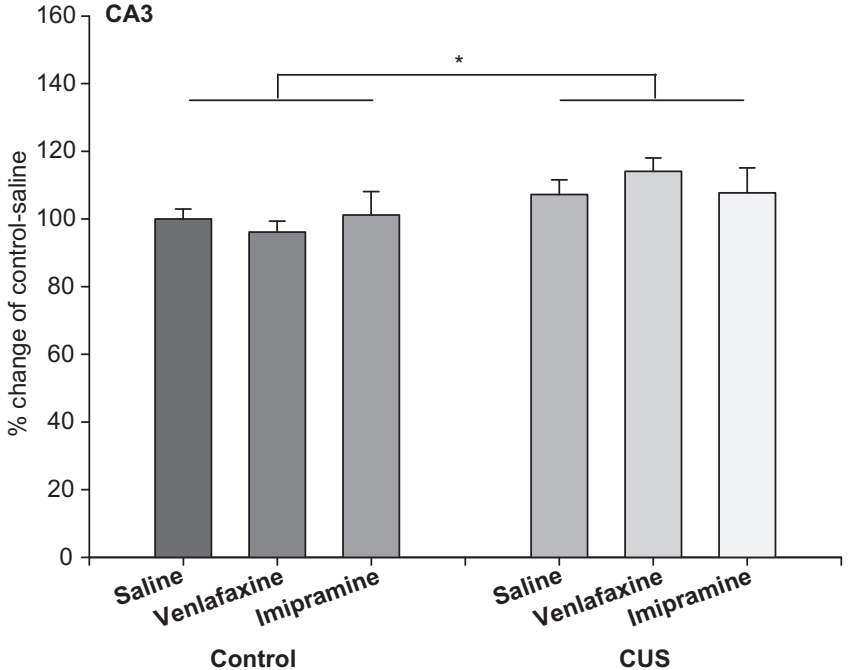

Fig. 6. Effects of CUS and chronic treatment with venlafaxine or imipramine on BDNF mRNA expression levels in the CA3 region of the ventral hippocampus. BDNF mRNA levels were significantly increased in animals exposed to CUS as compared to control animals. No effects of chronic antidepressant treatment were observed. Results are presented as mean \pm SEM. ${ }^{*} P<0.05$ (two-way ANOVA followed by Fishers LSD post hoc test).

\subsection{Correlations between basal anxiety levels and depressive-like behavior during CUS}

Correlation analyses of anxiety measures and depressive-like behaviors related to anhedonia revealed an overall significant positive correlation between the OF test (\% time in OF center) and saccharin consumption (per $100 \mathrm{~g}$ body weight) on day 18 $(r=0.343 ; P=0.014)$ and day $25(r=0.358 ; P=0.010)$; i.e., the lower the time spent in the center of the OF (conventionally interpreted as indicative of increased anxiety), the lower the saccharin consumption (conventionally interpreted as indicative of anhedonia). A trend towards a significant correlation was also observed on day $11(r=0.266 ; P=0.062)$. In addition, we found a significant negative correlation between the \% time animals spent touching the novel object and the time animals spent floating in the forced swim test $(r=-0.522 ; P=0.009)$; i.e., the lower the exploration (indicative of higher behavioral inhibition), the higher the floating behavior (conventionally interpreted as indicative of increased despair and depression-like behavior). All other correlations between the behavioral parameters examined pre- and postCUS were not significant (data not shown).

\section{Discussion}

The goal of the present study was to directly compare the effects on BDNF mRNA expression in the hippocampus with the 
behavioral effects seen after chronic treatment with different antidepressants in the chronic unpredictable stress model of depression. Rats exposed to CUS showed a significant decrease in body weight gain together with a reduced intake of a sweet (saccharin) solution compared to non-stressed rats. Importantly, chronic antidepressant treatment prevented the stress-induced reduction in the intake of a saccharin solution and reduced depressive-like behavior in the forced swim test. Chronic antidepressant treatment induced a significant up-regulation of BDNF mRNA expression in the GCL of the dorsal hippocampus independently of exposure to CUS. No effects of antidepressant treatment were observed in the dorsal or ventral hippocampal CA3 region. Interestingly, basal BDNF mRNA expression in the GCL of the dorsal hippocampus and in the CA3 region of the ventral hippocampus was increased rather than decreased after CUS.

The CUS model is a widely used model of depression which has been shown to induce several physiological and behavioral depressive-like symptoms (Willner, 2005). There are important individual differences in the vulnerability to stress, with strong evidence indicating that the high anxiety personality trait is a risk factor to develop depression (Jakobsson et al., 2008; Sandi, 2008; Sandi and Richter-Levin, 2009). In the present study, rats were tested in diverse tests for their natural tendencies to explore a variety of novel environments, a procedure that allows characterizing individuals' variation in basal (i.e., trait-like) anxiety levels before CUS exposure. This allowed questioning whether individual anxiety levels were correlated with subsequent depressive-like behavior. We found evidence in two independent tests of depression-like behaviors (saccharin consumption and forced swim test) for a correlation with animals' basal anxiety levels, suggesting that individuals with higher basal, trait-like anxiety levels are more prone to develop depressive-like symptoms when exposed to chronic stress.

The validation of our experimental protocol as an effective chronic stress-induction model was determined through its effects on body weight gain and on a behavioral measure of reward sensitivity. Decreased body weight is a well described symptom of exposure to chronic stress (Konkle et al., 2003; Lucca et al., 2008). In our study, CUS significantly decreased body weight gain and, as expected, this effect was not prevented by antidepressant treatment (Bekris et al., 2005; Song et al., 2006). Indeed, chronic treatment with imipramine further reduced body weight gain, an effect that was previously described specifically for treatment with imipramine at similar doses (Hughes and Pither, 1987; Mogensen et al., 1994). Previous work has shown that CUS reduces the intake of a sweet solution reflecting one of the core symptoms of depression, anhedonia, which was shown to be reversed by antidepressant treatment (Bekris et al., 2005; Jayatissa et al., 2006; Muscat and Willner, 1992; Papp et al., 1996). In agreement with the literature, rats exposed to our CUS protocol showed decreased consumption of and decreased preference for a sweet saccharin solution as compared to non-stressed rats, and this effect was prevented by chronic antidepressant treatment.

The forced swim test is widely used to predict antidepressant activity (Porsolt et al., 1978). In the current study, CUS animals were exposed to a modified forced swim test consisting of a single session that was used as a stressor towards the end of the CUS protocol (day 27), which allowed for the evaluation of potential effects of the antidepressant treatments in this behavioral test. In this test, rats typically react by initially displaying active coping behaviors (e.g., swimming, climbing) that might eventually turn into a floating behavior interpreted as learned helplessness or behavioral despair. Under basal conditions, floating behavior is decreased by antidepressant treatment (Porsolt et al., 1978). CUS has previously been reported to induce floating behavior in this test and it appears that this effect on floating is associated with CUS-induced anhedonia (Strekalova et al., 2004; Vitale et al., 2009). In our study, ani- mals treated chronically with imipramine or venlafaxine spent significantly less time floating as compared to saline treated animals. Interestingly, imipramine and venlafaxine induced different climbing and swimming behaviors. It has been reported that antidepressants with a noradrenergic profile enhance the climbing behavior whereas antidepressants with a serotonergic profile increase swimming (Detke et al., 1995). In line with this, an increase in climbing behavior was observed after treatment with imipramine, which has a more pronounced effect on the noradrenergic neurotransmission (Jordan et al., 1994), whereas venlafaxine, which affects both the serotonergic- and noradrenergic neurotransmitter system (Dawson et al., 1999), increased swimming. Thus, the present results show that chronic treatment with antidepressants inhibits depressive-like behavior in the forced swim test.

The mechanisms underlying depression as well as antidepressant activity are still not fully understood (Krishnan and Nestler, 2008; Sandi and Richter-Levin, 2009). Recently, a novel hypothesis has emerged suggesting a role for neurotrophic factors and subsequent structural and neuroplastic changes in the pathogenesis of depression and in its treatment (Castren, 2005; Nestler et al., 2002). Stressful life events are known to precipitate depressive illness in vulnerable persons (Caspi et al., 2003; Kendler et al., 2001) and stressful paradigms such as CUS have long been used to model depression. Notably, dendritic atrophy, cell loss and decreased BDNF levels in the hippocampus have been observed in response to different types of stressors (Nibuya et al., 1999; Rasmusson et al., 2002; Smith et al., 1995; Xu et al., 2004), and the effect of stress on BDNF was shown to be inhibited by antidepressant treatment (Nibuya et al., 1995).

In this study an increase, rather than a decrease, in BDNF mRNA levels was observed after CUS in the GCL of the dorsal hippocampus and in the CA3 region of the ventral hippocampus. However, since our study only included chronic, but not acute stress conditions, a note of caution should be added when linking these observations with the chronic nature of the stress procedure used. Exposure to CUS has been reported to reduce BDNF expression in the rat hippocampus (Grønli et al., 2006; Song et al., 2006). However, nowadays, the reported effects of CUS are contentious as several studies also have reported no effects or even increased BDNF levels in the hippocampus following exposure to chronic stress (Allaman et al., 2008; Bergstrom et al., 2008; Lee et al., 2006; Lucca et al., 2008; Schulte-Herbruggen et al., 2009; Vitale et al., 2009). It is possible that the discrepancy in the effect of CUS on BDNF is due to differences in the stress protocols applied in the different studies. Indeed, the effects of stress on BDNF mRNA expression in the hippocampus appear to be dependent on several factors such as the type of stressor, the intensity, the duration, the frequency and the number of exposures (Anisman and Matheson, 2005; Nair et al., 2006). It is important to emphasize that many of the studies showing a robust decrease of BDNF levels in the hippocampus have used severe stress protocols, such as immobilization stress, which have limited face validity with regard to the human depressive disorder. Moreover, regulation of BDNF in the hippocampus of depressed patients still needs to be revealed. Today, most human studies describe changes in serum BDNF levels in depressed patients; however, a clear correlation between serum and brain BDNF levels still needs to be established. Although speculative, it is possible that exposure to some stressful situations could activate mechanisms such as increased BDNF and hence increased BDNF mRNA expression in the hippocampus might reflect an adaptive protective response to the unpredictable stressors.

The present study shows no correlation between CUS-induced anhedonia and decreased BDNF mRNA levels in the hippocampus. It has been suggested that BDNF levels in the brain may not simply correlate with depression as no depressive-like behavior or increased vulnerability to develop stress-induced depressive-like 
behavior has been observed after reduction of BDNF levels or BDNF signaling (Duman and Monteggia, 2006; Ibarguen-Vargas et al., 2009; Saarelainen et al., 2003). Indeed, the current data suggest that BDNF is not per se involved in eliciting depressive-like behavior but support a role for BDNF in mediating aspects of antidepressant activity. The present data show that chronic treatment with different antidepressant drugs prevents CUS-induced anhedonic behavior and at the same time increases BDNF mRNA expression in the GCL of the dorsal hippocampus. The current study investigates the chronic but not the acute effects of antidepressant treatment. However, acute treatment with imipramine, venlafaxine and other antidepressants has previously been shown to have no effect on BDNF mRNA expression in the hippocampus (Coppell et al., 2003; De Foubert et al., 2004; Larsen et al., 2008; Nibuya et al., 1995).

Interestingly, BDNF mRNA expression in the hippocampus is regulated in the same direction by CUS and antidepressant treatment. It has recently been hypothesized that both depression and antidepressant activity develops as a consequence of remodeling of neuronal networks. Thus, BDNF could play an essential role in opening up these systems allowing activity-dependent plasticity and remodeling of these networks to take place; the function of which determine whether the plastic changes produce a depression- or antidepressant-like response (Castren et al., 2007). Interestingly, a similar example has also been revealed for the neuroplasticity molecule PSA-NCAM (i.e., the polysialylated form of the neural cell adhesion molecule), with both chronic stress (Cordero et al., 2005) and antidepressants treatment (Varea et al., 2007) inducing a reduction in its expression levels in the amygdala.

To our knowledge, this is the first study that directly compares the effects of CUS and antidepressant treatment on depressive-like symptoms together with the effects on BDNF mRNA expression in the dorsal and ventral hippocampus. Our data imply that regulation of BDNF in behavioral models of depression is complex and that BDNF mRNA expression in the hippocampus can be up-regulated despite the presence of depressive-like behavior. Hence, the results obtained do not support a simple link between depression and brain BDNF levels. In contrast, chronic antidepressant treatment normalized CUS induced depressive-like behavior and increased BDNF mRNA expression in the GCL of the dorsal hippocampus independently of exposure to CUS. Taken together, these results strongly support a role for BDNF in the mechanisms underlying antidepressant treatment.

\section{Role of funding source}

This study was financially supported by the Ministry for Technology and Innovation in Denmark and by grants from the EU 7th (FP7-HEALTH-F2M-2007-201600; MemStick) FP, the Swiss National Science Foundation (310000-120791), and Købmand i Odense Johann og Hanne Weimann f. Seedorffs Legat. The funding sources had no further roles in the study design; in the collection, analysis and interpretation of data; in the writing of the report, or in the decision to submit the paper for publication.

\section{Contributors}

All authors were involved in the design of the study. C.S. and M.H.L. designed the experimental protocol, supervised and performed the behavioral tests. In situ data were obtained by J.D.M. and M.H.L. M.H.L. undertook the statistical analyses and wrote the first draft of the manuscript. All authors contributed to and have approved the final manuscript.

\section{Conflict of interest statement}

None of the authors have had any financial, personal or other relationships with other people or organizations within 3 years of beginning the work submitted that could inappropriately influence, or perceive to influence, their work.

\section{Acknowledgments}

The authors would like to thank Jorge E. Castro Cifuentes for his help with the chronic unpredictable stress procedure. In addition, the authors would like to thank Pia R. Sandholm and Tine Engelbrecht for skillful technical assistance. This study was supported by the Ministry for Technology and Innovation in Denmark and by grants from the EU 7th (FP7-HEALTH-F2M-2007-201600; MemStick) FP, the Swiss National Science Foundation (310000120791), and Købmand i Odense Johann og Hanne Weimann f. Seedorffs Legat.

\section{References}

Aguilera M, Arias B, Wichers M, Barrantes-Vidal N, Moya J, Villa H, et al. Early adversity and 5-HTT/BDNF genes: new evidence of gene-environment interactions on depressive symptoms in a general population. Psychological Medicine 2009;1:8.

Allaman I, Papp M, Kraftsik R, Fiumelli H, Magistretti PJ, Martin JL. Expression of brain-derived neurotrophic factor is not modulated by chronic mild stress in the rat hippocampus and amygdala. Pharmacological Reports 2008;60:1001-7. Anisman H, Matheson K. Stress, depression, and anhedonia: caveats concerning animal models. Neuroscience and Biobehavioral Reviews 2005:29:525-46.

Aydemir O, Deveci A, Taneli F. The effect of chronic antidepressant treatment on serum brain-derived neurotrophic factor levels in depressed patients: a preliminary study. Progress in Neuro-Psychopharmacology \& Biological Psychiatry 2005;29:261-5.

Aydemir C, Yalcin ES, Aksaray S, Kisa C, Yildirim SG, Uzbay T, et al. Brain-derived neurotrophic factor (BDNF) changes in the serum of depressed women. Progress in Neuro-Psychopharmacology \& Biological Psychiatry 2006;30:1256-60.

Bannerman DM, Rawlins JN, McHugh SB, Deacon RM, Yee BK, Bast T, et al. Regional dissociations within the hippocampus-memory and anxiety. Neuroscience and Biobehavioral Reviews 2004;28:273-83.

Bekris S, Antoniou K, Daskas S, Papadopoulou-Daifoti Z. Behavioural and neurochemical effects induced by chronic mild stress applied to two different rat strains. Behavioural Brain Research 2005;161:45-59.

Bergstrom A, Jayatissa MN, Mork A, Wiborg 0 . Stress sensitivity and resilience in the chronic mild stress rat model of depression; an in situ hybridization study. Brain Research 2008;1196:41-52.

Brunoni AR, Lopes M, Fregni F. A systematic review and meta-analysis of clinical studies on major depression and BDNF levels: implications for the role of neuroplasticity in depression. The International Journal of Neuropsychopharmacology 2008;11:1169-80.

Caspi A, Sugden K, Moffitt TE, Taylor A, Craig IW, Harrington H, et al. Influence of life stress on depression: moderation by a polymorphism in the 5-HTT gene. Science 2003;301:386-9.

Castren E. Is mood chemistry? Nature Reviews. Neuroscience 2005;6:241-6.

Castren E, Voikar V, Rantamaki T. Role of neurotrophic factors in depression. Current Opinion in Pharmacology 2007;7:18-21.

Coppell AL, Pei Q, Zetterstrom TS. Bi-phasic change in BDNF gene expression following antidepressant drug treatment. Neuropharmacology 2003;44:903-10.

Cordero MI, Rodriguez JJ, Davies HA, Peddie CJ, Sandi C, Stewart MG. Chronic restraint stress down-regulates amygdaloid expression of polysialylated neural cell adhesion molecule. Neuroscience 2005;133:903-10.

Dawson LA, Nguyen HQ, Geiger A. Effects of venlafaxine on extracellular concentrations of 5-HT and noradrenaline in the rat frontal cortex: augmentation via 5-HT1A receptor antagonism. Neuropharmacology 1999;38:1153-63.

De Foubert G, Carney SL, Robinson CS, Destexhe EJ, Tomlinson R, Hicks CA, et al. Fluoxetine-induced change in rat brain expression of brain-derived neurotrophic factor varies depending on length of treatment. Neuroscience 2004;128:597-604.

Detke MJ, Rickels M, Lucki I. Active behaviors in the rat forced swimming test differentially produced by serotonergic and noradrenergic antidepressants. Psychopharmacology (Berl) 1995;121:66-72.

Drevets WC, Price JL, Furey ML. Brain structural and functional abnormalities in mood disorders: implications for neurocircuitry models of depression. Brain Structure \& Function 2008;213:93-118.

Duman RS, Monteggia LM. A neurotrophic model for stress-related mood disorders. Biological Psychiatry 2006;59:1116-27. 
Fendt M, Endres T. 2,3,5-Trimethyl-3-thiazoline (TMT), a component of fox odor just repugnant or really fear-inducing? Neuroscience and Biobehavioral Reviews 2008;32:1259-66.

Gervasoni N, Aubry JM, Bondolfi G, Osiek C, Schwald M, Bertschy G, et al. Partial normalization of serum brain-derived neurotrophic factor in remitted patients after a major depressive episode. Neuropsychobiology 2005;51:234-8.

Gonul AS, Akdeniz F, Taneli F, Donat O, Eker C, Vahip S. Effect of treatment on serum brain-derived neurotrophic factor levels in depressed patients. European Archives of Psychiatry and Clinical Neuroscience 2005;255:381-6.

Grønli J, Bramham C, Murison R, Kanhema T, Fiske E, Bjorvatn B, et al. Chronic mild stress inhibits BDNF protein expression and CREB activation in the dentate gyrus but not in the hippocampus proper. Pharmacology, Biochemistry, and Behavior 2006;85:842-9.

Herrero AI, Sandi C, Venero C. Individual differences in anxiety trait are related to spatial learning abilities and hippocampal expression of mineralocorticoid receptors. Neurobiology of Learning and Memory 2006;86:150-9.

Hughes RN, Pither JM. Chronic imipramine effects on exploratory behavior in rats. Pharmacology, Biochemistry, and Behavior 1987;27:359-62.

Ibarguen-Vargas Y, Surget A, Vourc'h P, Leman S, Andres CR, Gardier AM, et al. Deficit in BDNF does not increase vulnerability to stress but dampens antidepressant-like effects in the unpredictable chronic mild stress. Behavioural Brain Research 2009;202:245-51.

Jakobsson J, Cordero MI, Bisaz R, Groner AC, Busskamp V, Bensadoun JC, et al. KAP1 mediated epigenetic repression in the forebrain modulates behavioral vulnerability to stress. Neuron 2008;60:818-31.

Jayatissa MN, Bisgaard C, Tingstrom A, Papp M, Wiborg O. Hippocampal cytogenesis correlates to escitalopram-mediated recovery in a chronic mild stress rat model of depression. Neuropsychopharmacology 2006;31:2395-404.

Jordan S, Kramer GL, Zukas PK, Moeller M, Petty F. In vivo biogenic amine efflux in medial prefrontal cortex with imipramine, fluoxetine, and fluvoxamine. Synapse 1994;18:294-7.

Kendler KS, Thornton LM, Gardner CO. Genetic risk, number of previous depressive episodes, and stressful life events in predicting onset of major depression. The American Journal of Psychiatry 2001;158:582-6.

Kjelstrup KG, Tuvnes FA, Steffenach HA, Murison R, Moser EI, Moser MB. Reduced fear expression after lesions of the ventral hippocampus. Proceedings of the National Academy of Sciences of the United States of America 2002;99:10825-30.

Konkle AT, Baker SL, Kentner AC, Barbagallo LS, Merali Z, Bielajew C. Evaluation of the effects of chronic mild stressors on hedonic and physiological responses: sex and strain compared. Brain Research 2003;992:227-38.

Krishnan V, Nestler EJ. The molecular neurobiology of depression. Nature 2008;455:894-902.

Larsen MH, Olesen M, Woldbye DP, Hay-Schmidt A, Hansen HH, Ronn LC, Mikkelsen JD. Regulation of activity-regulated cytoskeleton protein (Arc) mRNA after acute and chronic electroconvulsive stimulation in the rat. Brain Research 2005;1064:161-5.

Larsen MH, Hay-Schmidt A, Ronn LC, Mikkelsen JD. Temporal expression of brainderived neurotrophic factor (BDNF) mRNA in the rat hippocampus after treatment with selective and mixed monoaminergic antidepressants. European Journal of Pharmacology 2008;578:114-22.

Lee KJ, Kim SJ, Kim SW, Choi SH, Shin YC, Park SH, et al. Chronic mild stress decreases survival, but not proliferation, of new-born cells in adult rat hippocampus. Experimental \& Molecular Medicine 2006;38:44-54.

Lucca G, Comim CM, Valvassori SS, Pereira JG, Stertz L, Gavioli EC, et al. Chronic mild stress paradigm reduces sweet food intake in rats without affecting brain derived neurotrophic factor protein levels. Current Neurovascular Research 2008;5:207-13.

Mogensen J, Pedersen TK, Holm S. Effects of chronic imipramine on exploration, locomotion, and food/water intake in rats. Pharmacology, Biochemistry, and Behavior 1994;47:427-35.

Monteggia LM, Barrot M, Powell CM, Berton O, Galanis V, Gemelli T, et al. Essential role of brain-derived neurotrophic factor in adult hippocampal function. Proceedings of the National Academy of Sciences of the United States of America 2004:101:10827-32.

Monteggia LM, Luikart B, Barrot M, Theobold D, Malkovska I, Nef S, et al. Brainderived neurotrophic factor conditional knockouts show gender differences in depression-related behaviors. Biological Psychiatry 2007;61:187-97.

Moser E, Moser MB, Andersen P. Spatial learning impairment parallels the magnitude of dorsal hippocampal lesions, but is hardly present following ventral lesions. The Journal of Neuroscience 1993;13:3916-25.

Muscat R, Willner P. Suppression of sucrose drinking by chronic mild unpredictable stress: a methodological analysis. Neuroscience and Biobehavioral Reviews 1992;16:507-17.

Nair A, Vadodaria KC, Banerjee SB, Benekareddy M, Dias BG, Duman RS, et al. Stressor-specific regulation of distinct brain-derived neurotrophic factor transcripts and cyclic AMP response element-binding protein expression in the postnatal and adult rat hippocampus. Neuropsychopharmacology 2006.

Nestler EJ, Barrot M, DiLeone RJ, Eisch AJ, Gold SJ, Monteggia LM. Neurobiology of depression. Neuron 2002;34:13-25.

Nibuya M, Morinobu S, Duman RS. Regulation of BDNF and trkB mRNA in rat brain by chronic electroconvulsive seizure and antidepressant drug treatments. The Journal of Neuroscience 1995;15:7539-47.
Nibuya M, Takahashi M, Russell DS, Duman RS. Repeated stress increases catalytic TrkB mRNA in rat hippocampus. Neuroscience Letters 1999;267:81-4.

Papp M, Moryl E, Willner P. Pharmacological validation of the chronic mild stress model of depression. European Journal of Pharmacology 1996;296:129-36.

Paxinos G, Watson C. The rat brain in stereotaxic coordinates. London: Academic Press Ltd.; 1998.

Porsolt RD, Anton G, Blavet N, Jalfre M. Behavioural despair in rats: a new model sensitive to antidepressant treatments. European Journal of Pharmacology 1978;47:379-91.

Rasmusson AM, Shi L, Duman R. Downregulation of BDNF mRNA in the hippocampal dentate gyrus after re-exposure to cues previously associated with footshock. Neuropsychopharmacology 2002;27:133-42.

Rogoz Z, Skuza G, Legutko B. Repeated treatment with mirtazepine induces brainderived neurotrophic factor gene expression in rats. Journal of Physiology and Pharmacology 2005;56:661-71.

Saarelainen T, Hendolin P, Lucas G, Koponen E, Sairanen M, MacDonald E, et al. Activation of the TrkB neurotrophin receptor is induced by antidepressant drugs and is required for antidepressant-induced behavioral effects. The Journal of Neuroscience 2003;23:349-57.

Sandi C. Adding complexity to emotion-cognition interactions: the stressed individual. Frontiers in Neuroscience 2008;2:134-5.

Sandi C, Richter-Levin G. From high anxiety trait to depression: a neurocognitive hypothesis. Trends in Neuroscience 2009.

Sandi C, Cordero MI, Ugolini A, Varea E, Caberlotto L, Large CH. Chronic stressinduced alterations in amygdala responsiveness and behavior-modulation by trait anxiety and corticotropin-releasing factor systems. The European Journal of Neuroscience 2008;28:1836-48.

Schulte-Herbruggen O, Fuchs E, Abumaria N, Ziegler A, Danker-Hopfe H, Hiemke C, et al. Effects of escitalopram on the regulation of brain-derived neurotrophic factor and nerve growth factor protein levels in a rat model of chronic stress. Journal of Neuroscience Research 2009;87:2551-60.

Schumacher J, Jamra RA, Becker T, Ohlraun S, Klopp N, Binder EB, et al. Evidence for a relationship between genetic variants at the brain-derived neurotrophic factor (BDNF) locus and major depression. Biological Psychiatry 2005;58:307-14

Shirayama Y, Chen AC, Nakagawa S, Russell DS, Duman RS. Brain-derived neurotrophic factor produces antidepressant effects in behavioral models of depression. The Journal of Neuroscience 2002;22:3251-61.

Sillaber I, Panhuysen M, Henniger MS, Ohl F, Kuhne C, Putz B, et al. Profiling of behavioral changes and hippocampal gene expression in mice chronically treated with the SSRI paroxetine. Psychopharmacology (Berl) 2008;200:557-72.

Siuciak JA, Lewis DR, Wiegand SJ, Lindsay RM. Antidepressant-like effect of brainderived neurotrophic factor (BDNF). Pharmacology, Biochemistry, and Behavior 1997;56:131-7

Smith MA, Makino S, Kvetnansky R, Post RM. Stress and glucocorticoids affect the expression of brain-derived neurotrophic factor and neurotrophin-3 mRNAs in the hippocampus. The Journal of Neuroscience 1995;15:1768-77.

Song L, Che W, Min-Wei W, Murakami Y, Matsumoto K. Impairment of the spatial learning and memory induced by learned helplessness and chronic mild stress. Pharmacology, Biochemistry, and Behavior 2006;83:186-93.

Strekalova T, Spanagel R, Bartsch D, Henn FA, Gass P. Stress-induced anhedonia in mice is associated with deficits in forced swimming and exploration. Neuropsychopharmacology 2004;29:2007-17.

Trivedi MA, Coover GD. Lesions of the ventral hippocampus, but not the dorsal hippocampus, impair conditioned fear expression and inhibitory avoidance on the elevated T-maze. Neurobiology of Learning Memory 2004;81:172-84.

Varea E, Castillo-Gomez E, Gomez-Climent MA, Blasco-Ibanez JM, Crespo C Martinez-Guijarro FJ, et al. Chronic antidepressant treatment induces contrasting patterns of synaptophysin and PSA-NCAM expression in different regions of the adult rat telencephalon. European Neuropsychopharmacology 2007

Vitale G, Ruggieri V, Filaferro M, Frigeri C, Alboni S, Tascedda F, et al. Chronic treatment with the selective NOP receptor antagonist [Nphe(1), Arg (14), Lys (15)]N/OFQ-NH (2) (UFP-101) reverses the behavioural and biochemical effects of unpredictable chronic mild stress in rats. Psychopharmacology (Berl) 2009;207:173-89.

Wichers M, Kenis G, Jacobs N, Mengelers R, Derom C, Vlietinck R, et al. The BDNF $\operatorname{Val}(66)$ Met $\times 5$-HTTLPR $\times$ child adversity interaction and depressive symptoms: an attempt at replication. American Journal of Medical Genetics. Part B, Neuropsychiatric Genetics 2008;147B:120-3.

Willner P. Validity, reliability and utility of the chronic mild stress model of depression: a 10-year review and evaluation. Psychopharmacology (Berl) 1997;134:319-29.

Willner P. Chronic mild stress (CMS) revisited: consistency and behaviouralneurobiological concordance in the effects of CMS. Neuropsychobiology 2005;52:90-110.

Xu H, Luo C, Richardson JS, Li XM. Recovery of hippocampal cell proliferation and BDNF levels, both of which are reduced by repeated restraint stress, is accelerated by chronic venlafaxine. The Pharmacogenomics Journal 2004;4:322-31. 